\title{
ETA INVARIANTS WITH SPECTRAL BOUNDARY CONDITIONS
}

\author{
P. GILKEY, K. KIRSTEN, AND J. H. PARK
}

\begin{abstract}
We study the asymptotics of the heat trace $\operatorname{Tr}\left\{f P e^{-t P^{2}}\right\}$ where $P$ is an operator of Dirac type, where $f$ is an auxiliary smooth smearing function which is used to localize the problem, and where we impose spectral boundary conditions. Using functorial techniques and special case calculations, the boundary part of the leading coefficients in the asymptotic expansion is found.
\end{abstract}

\section{INTRODUCTION}

Let $P$ be an operator of Dirac type with leading symbol $\gamma$ on a vector bundle $V$ over a compact $m$ dimensional Riemannian manifold $M$ with smooth boundary $\partial M$. One may choose a Hermitian inner product $(\cdot, \cdot)$ and a Hermitian connection $\nabla$ on $V$ so that $\gamma$ is skew-adjoint and so that $\nabla \gamma=0$ [11; such structures are said to be compatible with the given Clifford module structure $\gamma$. Let indices $i, j$ range from 1 to $m$ and index a local orthonormal frame $\left\{e_{i}\right\}$ for the tangent bundle of $M$. We adopt the Einstein convention and sum over repeated indices to expand

$$
P=\gamma_{i} \nabla_{e_{i}}+\psi_{P}
$$

where $\psi_{P}$ is a smooth endomorphism of $V$; the sign convention for $\psi_{P}$ differs from that in [11, 12]. Note that the matrices $\gamma_{i}$ are skew-adjoint endomorphisms of $V$ satisfying the Clifford commutation relations

$$
\gamma_{i} \gamma_{j}+\gamma_{j} \gamma_{i}=-2 \delta_{i j}
$$

If $\partial M$ is non-empty, then we must impose suitable boundary conditions. For $m$ even, $P$ always admits local elliptic boundary conditions; see, for example, the discussion of bag boundary conditions in [7. 8. However, if $m$ is odd, there is a topological obstruction to the existence of local boundary conditions for certain operators. We therefore introduce spectral boundary conditions; these boundary conditions, which are defined regardless of the parity of $m$, play a crucial role in the index theorem for manifolds with boundary [3].

Spectral boundary conditions were first introduced by Atiyah et. al. 3 in their study of Hirzebruch signature theorem for manifolds with non-empty boundary. The crucial point at issue was the definition of a suitable elliptic boundary value problem for the signature operator whose index was the signature of the manifold. Although the de Rham complex, whose index is the Euler characteristic, admits local boundary conditions (i.e. boundary conditions which are a mixture of Robin and Dirichlet), the signature complex does not. The signature complex does admit spectral boundary conditions - these are pseudo-differential boundary conditions and their introduction was a crucial turning point.

In order to describe these boundary conditions, near the boundary we choose a local orthonormal frame so $e_{m}$ is the inward unit geodesic normal vector field and

Key words and phrases. spectral boundary conditions, operator of Dirac type, heat equation, eta invariant

2000 Mathematics Subject Classification. 58J50. 
$\left\{e_{a}\right\}$ for $1 \leq a \leq m-1$ is the induced orthonormal frame for the tangent bundle of the boundary. Let

$$
\gamma_{a}^{T}:=-\gamma_{m} \gamma_{a}
$$

be the induced tangential Clifford module structure. Let $\psi_{A}$ be an auxiliary smooth endomorphism of $\left.V\right|_{\partial M}$. Consider the auxiliary operator of Dirac type on $\left.V\right|_{\partial M}$

$$
A:=\gamma_{a}^{T} \nabla_{e_{a}}+\psi_{A} .
$$

Assume $A$ has no purely imaginary eigensections. Let $C$ be a suitable contour in the complex plane containing the spectrum of $A$ with positive real part. Let

$$
\Pi_{A}^{+}:=\frac{1}{2 \pi \sqrt{-1}} \int_{C}(A-\lambda)^{-1} d \lambda
$$

be the spectral boundary operator; $\Pi_{A}^{+}$is spectral projection on the generalized eigenspaces associated to eigenvalues with positive real part. Let $P_{A}$ be the realization of $P$ with respect to the boundary conditions defined by $\Pi_{A}^{+}$.

The spectral information regarding this boundary value problem is encoded in the zeta function and the eta function which are defined as follows. Assume for the sake of simplicity that $P_{A}$ is self-adjoint (we will be forced to drop this requirement presently). Let $\left(\lambda_{l}, \varphi_{l}\right)$ be a spectral resolution of $P_{A} ;\left\{\varphi_{l}\right\}$ is a complete orthonormal basis for $L^{2}(V)$ such that

$$
P_{A} \varphi_{l}=\lambda_{l} \varphi_{l},\left.\quad \Pi_{A}^{+} \varphi_{l}\right|_{\partial M}=0 .
$$

Then the zeta function associated with $P_{A}^{2}$ is,

$$
\zeta(s ; P, A):=\sum_{\lambda_{l} \neq 0}\left(\lambda_{l}^{2}\right)^{-s}
$$

valid for $\Re s>m / 2$. Note, the fact that $P_{A}$ as a first order differentiable operator can have positive and negative eigenvalues does not enter the zeta function of the Laplace-type operator $P_{A}^{2}$. However, the sign is taken into account defining the eta function of $P_{A}$,

$$
\eta(s ; P, A):=\sum_{\lambda_{l} \neq 0} \operatorname{sign}\left(\lambda_{l}\right)\left|\lambda_{l}\right|^{-s},
$$

valid for $\Re s>m-1$. Similarly, one can define $\zeta(s ; A)$ and $\eta(s ; A)$; since $\partial M$ is closed, there is no boundary condition required.

Although the above series representations (1.a) and (1.b) are valid only in the given region of the complex $s$-plane, the eta and zeta functions can be analytically continued to meromorphic functions defined on the whole complex plane. The value $\eta(0 ; P, A)$ is essential for the description of the index of $P_{A}$.

One can also discuss the heat trace. Let $\phi$ be the 'initial temperature distribution' and let $u_{\phi}(t, x)$ denote the subsequent temperature distribution. Then $u_{\phi}(t, x)$ is determined by the equations

$$
\left(\partial_{t}+P^{2}\right) u_{\phi}(t, x)=0,\left.\quad \Pi_{A}^{+} u_{\phi}\right|_{\partial M}=0 \quad \text { and } \quad u_{\phi}(0, x)=\phi(x) .
$$

The associated fundamental solution $\mathcal{K}: \phi \rightarrow u_{\phi}$ is then given by $\mathcal{K}=e^{-t P_{A}^{2}}$. Let $d x$ and $d y$ be the Riemannian measures on $M$ and on $\partial M$ respectively. There exists a smooth endomorphism-valued kernel $K\left(t, x, \bar{x}, P^{2}, A\right): V_{\bar{x}} \rightarrow V_{x}$ such that

$$
u_{\phi}(t, x)=(\mathcal{K} \phi)(t, x)=\int_{M} K\left(t, x, \bar{x}, P^{2}, A\right) \phi(\bar{x}) d \bar{x} .
$$


For fixed $t$, the operator $\mathcal{K}(t): \phi \rightarrow \phi(t, \cdot)$ is of trace class. For $F \in C^{\infty}(\operatorname{End}(V))$ a smooth auxiliary smearing endomorphism used for localizing the problem, we define

$$
\begin{aligned}
& a^{\zeta}(F, P, A):=\operatorname{Tr}_{L^{2}}\left(F e^{-t P_{A}^{2}}\right)=\int_{M} \operatorname{Tr}_{V_{x}}\left(F(x) K\left(t, x, x, P^{2}, A\right)\right) d x \\
& a^{\eta}(F, P, A):=\operatorname{Tr}_{L^{2}}\left(F P_{A} e^{-t P_{A}^{2}}\right)=\int_{M} \operatorname{Tr}_{V_{x}}\left(F(x) P_{A} K\left(t, x, x, P^{2}, A\right)\right) d x .
\end{aligned}
$$

Grubb and Seeley [25] showed that there are asymptotic expansions as $t \downarrow 0^{+}$of the form:

$$
\begin{aligned}
& a^{\zeta}(F, P, A) \sim \sum_{n=0}^{m-1} a_{n}^{\zeta}(F, P, A) t^{(n-m) / 2}+\mathcal{O}(\ln t), \\
& a^{\eta}(F, P, A) \sim \sum_{n=0}^{m-1} a_{n}^{\eta}(F, P, A) t^{(n-m-1) / 2}+\mathcal{O}\left(t^{1 / 2} \ln t\right) .
\end{aligned}
$$

We refer to the coefficients $a_{n}^{\zeta}$ and $a_{n}^{\eta}$ as the zeta and eta invariants respectively.

We note that there are in fact full asymptotic series for $a^{\zeta}$ and $a^{\eta}$. However nonlocal terms and $\log$ terms arise when $n \geq m$. Since we shall assume that $n<m$, these terms play no role for us. We shall normally assume that $F=f$. Id where $f \in C^{\infty}(M)$ is scalar valued, but it will be convenient occasionally to have this more general setting available.

The Mellin transform can be used to relate the zeta and eta functions and the small- $t$ asymptotic expansion of the heat-trace. For $f=1$, one has [21, 41]

$$
\begin{aligned}
\operatorname{Res} \zeta\left(\frac{m-n}{2} ; P, A\right) & =\frac{a_{n}^{\zeta}(1, P, A)}{\Gamma\left(\frac{m-n}{2}\right)}, \\
\operatorname{Res} \eta(m-n ; P, A) & =\frac{2 a_{n}^{\eta}(1, P, A)}{\Gamma\left(\frac{m-n+1}{2}\right)} .
\end{aligned}
$$

Similar formulas hold for general endomorphism $F$; this will play an important role in our subsequent development.

The heat trace coefficients $a_{n}^{\zeta}$ and $a_{n}^{\eta}$ of Equation 1.c are locally computable for $n<m$; they play a crucial role in many areas. For example, the particular coefficient $a_{m}^{\zeta}$ is relevant in the quantum mechanics of closed cosmologies, where it describes how quantum effects modify the behaviour of the universe near classical singularities [14] 18, 40]. More generally, the leading coefficients $a_{n}^{\zeta}, n=0,1, \ldots, m$ are needed in different quantum field theories. These theories are generically plagued by divergences which are removed by a renormalization. In the zeta function scheme [17, as well as in the framework of recent developments of algebraic quantum field theory [33, at one-loop, divergences are completely described by the leading coefficients. As a result, their knowledge is equivalent to a knowledge of the one-loop renormalization group equations [43, which provides one reason for the consideration of heat kernel coefficients in physics. In addition, if an exact evaluation of relevant quantities is not possible, asymptotic expansions are often very useful and most suitably given in terms of heat kernel coefficients [5] [15. In this context of quantum field theories, apart from cosmology, spectral boundary conditions most prominently make their appearance in bag models where they have important advantages over local elliptic boundary conditions. In particular, it is the only selfadjoint boundary condition which respects the charge conjugation property and the so-called $\gamma_{5}$ symmetry [19, 27, 34. In Euclidean gauge field theories, this property enables one to consider a compactified Dirac problem where spectral information such as functional determinants are directly related to the original problem 28, 42.

Whereas the above relates to $a_{n}^{\zeta}$, the $\eta$-function arises in the analysis of fermion number fractionization in different field theory models [35, 36, 39]. The fermion number $N$ is a transcendental function of the parameters of the theory and is related to $\eta(0 ; H, A)$ of the pertinent Dirac Hamiltonian $H$ and boundary operator 
$A$. In a simplified consideration 30 the fermion number of the vacuum will be formally obtained by filling the Dirac sea,

$$
\begin{aligned}
N= & \text { number of negative-energy states of } H] \\
= & \frac{1}{2}\{[\text { (numb. of pos.-en. states of } \mathrm{H})+(\text { numb. of neg.-en. states of } \mathrm{H})] \\
& -[(\text { numb. of pos.-en. states of } \mathrm{H})-(\text { numb. of neg.-en. states of } \mathrm{H})]\}
\end{aligned}
$$

Regularizing this divergent expression it becomes $(1 / 2)[0-\eta(0 ; H, A)]$. A rigorous proof can be found in [31].

Furthermore, interpreting (1.a) and (1.b) as a moment problem for the spectral density function, even and odd part of the density can be found provided $\zeta(s ; H, A)$ and $\eta(s ; H, A)$ can be evaluated 37. Knowledge of the leading coefficients $a_{n}^{\zeta}$ respectively $a_{n}^{\eta}$ amounts to an asymptotic knowledge of the even and odd part of the density for large eigenvalues $\left|\lambda_{l}\right|$ opening up the possibility for the approximate evaluation of different quantities in quantum field theories as for example the finite temperature induced fermion number [36].

The invariants $a_{n}^{\zeta}$ have been studied extensively [16, 23, 25, 26]; the invariants $a_{n}^{\eta}$ have received a bit less attention. We may decompose

$$
\begin{aligned}
& a_{n}^{\zeta}(F, P, A)=a_{n}^{\zeta, M}(F, P)+a_{n}^{\zeta, \partial M}(F, P, A), \quad \text { and } \\
& a_{n}^{\eta}(F, P, A)=a_{n}^{\eta, M}(F, P)+a_{n}^{\eta, \partial M}(F, P, A)
\end{aligned}
$$

as the sum of interior and boundary contributions. There exist local endomorphism valued invariants $e_{n}^{\zeta, M}(x, P)$ and $e_{n}^{\eta, M}(x, P)$, which are homogeneous of weight $n$ in the jets of the total symbol of $P$, so that

$$
\begin{aligned}
& a_{n}^{\zeta, M}(F, P)=\int_{M} \operatorname{Tr}\left\{F(x) e_{n}^{\zeta, M}(x, P)\right\} d x, \quad \text { and } \\
& a_{n}^{\eta, M}(F, P)=\int_{M} \operatorname{Tr}\left\{F(x) e_{n}^{\eta, M}(x, P)\right\} d x .
\end{aligned}
$$

We note there is a parity constraint for the interior invariants;

$$
a_{n}^{\zeta, M}=0 \quad \text { if } n \quad \text { is odd and } a_{n}^{\eta, M}=0 \quad \text { if } n \text { is even. }
$$

Formulae for the invariants $a_{n}^{\zeta, M}$ for $n=0,2,4,6,8$ follow from work of [2, 4, 20, 32, similar formulae for the invariants $a_{n}^{\eta, M}$ are known for $n=1,3[11$.

Let $\nabla_{m}^{k} F$ denote the $k^{\text {th }}$ normal covariant derivative of the endomorphism $F$. There are local invariants $e_{n, k}^{\zeta, \partial M}(y, P, A)$ and $e_{n, k}^{\eta, \partial M}(y, P, A)$ which are homogeneous of weight $n-k-1$ in the jets of the total symbol of $P$ and of $A$ so that

$$
\begin{aligned}
& a_{n}^{\zeta, \partial M}(F, P, A)=\sum_{k<n} \int_{\partial M} \operatorname{Tr}\left\{\nabla_{m}^{k} F(y) \cdot e_{n, k}^{\zeta, \partial M}(y, P, A)\right\} d y \quad \text { and } \\
& a_{n}^{\eta, \partial M}(F, P, A)=\sum_{k<n} \int_{\partial M} \operatorname{Tr}\left\{\nabla_{m}^{k} F(y) \cdot e_{n, k}^{\eta, \partial M}(y, P, A)\right\} d y
\end{aligned}
$$

Let $\Omega_{i j}$ be the curvature of the connection $\nabla$. We define

$$
\begin{aligned}
W_{i j} & :=\Omega_{i j}-\frac{1}{4} R_{i j k l} \gamma_{k} \gamma_{\ell}, \quad \beta(m):=\Gamma\left(\frac{m}{2}\right) \Gamma\left(\frac{1}{2}\right)^{-1} \Gamma\left(\frac{m+1}{2}\right)^{-1}, \quad \text { and } \\
E & :=\frac{1}{2}\left(\psi_{P ; i} \gamma_{i}-\gamma_{i} \psi_{P ; i}\right)-\psi_{P}^{2}-\frac{1}{4}\left(\psi_{P} \gamma_{i}+\gamma_{i} \psi_{P}\right)\left(\psi_{P} \gamma_{i}+\gamma_{i} \psi_{P}\right) \\
& -\frac{1}{2} \gamma_{i} \gamma_{j} W_{i j}-\frac{1}{4} \tau .
\end{aligned}
$$

Let $\tau:=R_{i j j i}$ be the scalar curvature and let $L_{a b}$ be the second fundamental form. We can use [11, 16, 23] to see:

Theorem 1.1. If $P_{A}$ is self-adjoint, if $A$ is self-adjoint, and if $F=f \cdot I d$ is scalar,

(1) $a_{0}^{\zeta}(F, P, A)=(4 \pi)^{-m / 2} \int_{M} f \operatorname{Tr}\{\operatorname{Id}\} d x$.

(2) If $m \geq 2, a_{1}^{\zeta}(F, P, A)=(4 \pi)^{-(m-1) / 2} \frac{1}{4}(\beta(m)-1) \int_{\partial M} f \operatorname{Tr}\{\operatorname{Id}\} d y$.

(3) If $m \geq 3, a_{2}^{\zeta}(F, P, A)=(4 \pi)^{-m / 2} \int_{M} f \operatorname{Tr}\left\{\frac{1}{6} \tau \operatorname{Id}+E\right\} d x$ $+(4 \pi)^{-m / 2} \int_{\partial M}\left\{\frac{1}{3}\left(1-\frac{3}{4} \pi \beta(m)\right) L_{a a} f-\frac{m-1}{2(m-2)}\left(1-\frac{1}{2} \pi \beta(m)\right) f_{; m}\right\} \operatorname{Tr}\{\mathrm{Id}\} d y$. 
We refer to 23 for the corresponding computation of $a_{3}^{\zeta}(f, D, \mathcal{B})$. In this note, we establish formulas for $a_{n}^{\eta}$ without self-adjointness assumptions:

Theorem 1.2. Let $F=f \cdot$ Id be scalar, then

(1) $a_{0}^{\eta}(F, P, A)=0$.

(2) If $m \geq 2, a_{1}^{\eta}(F, P, A)=(4 \pi)^{-m / 2}(1-m) \int_{M} f \operatorname{Tr}\left\{\psi_{P}\right\} d x$.

(3) If $m \geq 3, a_{2}^{\eta}(F, P, A)=(4 \pi)^{-(m-1) / 2} \int_{\partial M} f \operatorname{Tr}\left\{\frac{2-m}{4}(\beta(m)-1) \psi_{P}\right.$

$\left.-\frac{1}{4} \beta(m) \gamma_{m} \psi_{A}\right\} d y$.

(4) If $m \geq 4, a_{3}^{\eta}(F, P, A)=-\frac{1}{12}(4 \pi)^{-m / 2} \int_{M} f \operatorname{Tr}\left\{\left[2(m-1) \psi_{P ; i}\right.\right.$

$\left.+3(4-m) \psi_{P} \gamma_{i} \psi_{P}+3 \gamma_{j} \psi_{P} \gamma_{j} \gamma_{i} \psi_{P}\right]_{; i}+(3-m)\left\{\tau \psi_{P}+6 \gamma_{i} \gamma_{j} W_{i j} \psi_{P}\right.$

$\left.\left.-6 \psi_{P} \psi_{P ; i} \gamma_{i}+(4-m) \psi_{P} \psi_{P} \psi_{P}+3 \psi_{P} \psi_{P} \gamma_{i} \psi_{P} \gamma_{i}\right\}\right\} d x$

$+(4 \pi)^{-m / 2} \int_{\partial M} \operatorname{Tr}\left\{\frac{(m-3)(m-1)}{2(m-2)}\left(1-\frac{1}{2} \pi \beta(m)\right) f_{; m} \psi_{P}\right.$

$-f \frac{(3-m)^{2}}{4(m-2)}\left(\psi_{P} \psi_{A}+\gamma_{m} \psi_{P} \gamma_{m} \psi_{A}\right)+f \frac{3-m}{3}\left(1-\frac{3}{4} \pi \beta(m)\right) L_{a a} \psi_{P}$

$+f\left\{\frac{(m-3)(m-1)}{2(m-2)}\left(1-\frac{1}{2} \pi \beta(m)\right)-\frac{1}{6}(m-1)\right\} \psi_{P ; m}$

$-f \frac{3-m}{4(m-2)}\left(\gamma_{a}^{T} \psi_{P} \gamma_{a}^{T} \psi_{A}-\gamma_{a} \psi_{P} \gamma_{a} \psi_{A}+2 \gamma_{m} \gamma_{a}^{T} \psi_{A} \gamma_{a}^{T} \psi_{A}\right)$

$\left.+\frac{1}{2(m-2)}\left(1-\frac{1}{2} \pi(m-1) \beta(m)\right)\left(\frac{m-3}{1-m} f L_{a a}+f_{; m}\right) \gamma_{m} \psi_{A}\right\} d y$.

As the interior integrands $a_{1}^{\eta, M}$ and $a_{3}^{\eta, M}$ were determined previously by Branson and Gilkey [1], we shall concentrate upon determining the boundary integrands.

Here is a brief outline to the paper. In Section 2 we derive some basic functorial properties of these invariants. One of the peculiarities of using the 'functorial approach' is that it is necessary to work in a very general context and then specialize subsequently. To employ this method, we will have to work with operators which are not self-adjoint despite the fact that the examples which arise in practice are usually self-adjoint. In Section[3] we express the invariants $a_{n}^{\eta, \partial M}$ in terms of a Weyl basis with certain undetermined coefficients and begin the evaluation of these coefficients. We complete the proof of Theorem [1.2] in Sections 4 and 5 by completing the determination of the coefficients.

\section{FunCtorial PRoperties}

We refer to 22, 23] for the proof of the following result which describes the adjoint structures:

Lemma 2.1. Let $P^{*}$ be the formal adjoint of $P$, let $A^{*}$ be the formal adjoint of $A$, and let $A^{\#}:=\gamma_{m} A^{*} \gamma_{m}$.

(1) The operator $A^{\#}$ defines the adjoint boundary condition for $P^{*}$.

(2) We have $\psi_{P^{*}}=\psi_{P}^{*}, \psi_{A^{*}}=\psi_{A}^{*}$, and $\psi_{A^{\#}}=\gamma_{m} \psi_{A}^{*} \gamma_{m}+L_{a a} \mathrm{Id}$.

(3) If $\psi_{P}$ is self-adjoint, and if $\psi_{A}=\gamma_{m} \psi_{A}^{*} \gamma_{m}+L_{a a}$ Id, then $P_{A}$ is self-adjoint on $L^{2}(V)$.

The next observation follows from work of Grubb and Seeley [26].

Lemma 2.2. Let $n<m$. Assume that the metric on $M$ is product near the boundary, that $P_{A}$ is self-adjoint, that $A$ is self-adjoint, and that the coefficients of $P$ and of $A$ are independent of the normal variable near the boundary. Let $F$ be an endomorphism of $V$ whose coefficients are independent of the normal variable near the boundary.

(1) If $n$ is even, then $a_{n}^{\zeta, \partial M}(F, P, A)=-\frac{1}{2(m-n) \Gamma\left(\frac{1}{2}\right)} a_{n-1}^{\eta}(F, A)$.

(2) If $n$ is odd, then $a_{n}^{\zeta, \partial M}(F, P, A)=\frac{1}{4}(\beta(m-n+1)-1) a_{n-1}^{\zeta}(F, A)$.

Taking the adjoint yields yet another useful property. 
Lemma 2.3. Let $n<m$. Let $(P, A)$ be real operators on a real bundle $V$. Suppose $V$ is equipped with a fiber metric. Let $P^{*}$ be the formal adjoint of $P$ and let $F^{*}$ be the adjoint of $F$. Set $A^{\#}=\gamma_{m} A^{*} \gamma_{m}$. Then $a_{n}^{\eta, \partial M}(F, P, A)=a_{n}^{\eta, \partial M}\left(F^{*}, P^{*}, A^{\#}\right)$.

Proof. As we are in the real setting, taking the complex conjugate plays no role. Consequently

$$
\operatorname{Tr}_{L^{2}}\left\{F P e^{-t P_{A}^{2}}\right\}=\operatorname{Tr}_{L^{2}}\left\{F^{*} P^{*} e^{-t\left(P_{A}^{*}\right)^{2}}\right\} .
$$

The Lemma follows by equating powers of $t$ in the asymptotic expansions and by using Lemma 2.1 to see that $A^{\#}$ defines the adjoint boundary condition.

There is a useful relation between the $\zeta$ and the $\eta$ invariants.

Lemma 2.4. Let $F \in C^{\infty}(\operatorname{End}(V))$. Let $(A, P)$ be as above and let $n<m$.

(1) Let $P_{\varepsilon}:=P+\varepsilon F$. Then

(a) $\partial_{\varepsilon} a_{n}^{\eta}\left(1, P_{\varepsilon}, A\right)=(n-m) a_{n-1}^{\zeta}\left(F, P_{\varepsilon}, A\right)$.

(b) $\partial_{\varepsilon} a_{n}^{\zeta}\left(1, P_{\varepsilon}, A\right)=-2 a_{n-1}^{\eta}\left(F, P_{\varepsilon}, A\right)$.

(2) Let $P_{\varepsilon}:=P+\varepsilon \mathrm{Id}$. Then

(a) $\partial_{\varepsilon} a_{n}^{\eta}\left(F, P_{\varepsilon}, A\right)=(n-m) a_{n-1}^{\zeta}\left(F, P_{\varepsilon}, A\right)$.

(b) $\partial_{\varepsilon} a_{n}^{\zeta}\left(F, P_{\varepsilon}, A\right)=-2 a_{n-1}^{\eta}\left(F, P_{\varepsilon}, A\right)$.

(3) Let $P_{\varepsilon}:=e^{-\varepsilon f} P$ where $f$ is a smooth scalar function vanishing on $\partial M$. Then $\partial_{\varepsilon} a_{n}^{\eta}\left(1, P_{\varepsilon}, A\right)=(m-n) a_{n}^{\eta}\left(f, P_{\varepsilon}, A\right)$.

Proof. To prove Assertion (1), let $P_{\varepsilon}:=P+\varepsilon F$. We compute

$$
\begin{aligned}
& \sum_{n} \partial_{\varepsilon} a_{n}^{\eta}\left(1, P_{\varepsilon}, A\right) t^{(n-m-1) / 2} \sim \partial_{\varepsilon} \operatorname{Tr}\left\{P_{\varepsilon} e^{\left.-t P_{\varepsilon, A}^{2}\right\}}\right. \\
= & \operatorname{Tr}\left\{F\left(\mathrm{Id}-2 t P_{\varepsilon}^{2}\right) e^{-t P_{\varepsilon, A}^{2}}\right\}=\left(1+2 t \partial_{t}\right) \operatorname{Tr}\left\{F e^{\left.-t P_{\varepsilon, A}^{2}\right\}}\right. \\
\sim & \left(1+2 t \partial_{t}\right) \sum_{k} a_{k}^{\zeta}\left(F, P_{\varepsilon}, A\right) t^{(k-m) / 2} \\
= & \sum_{k}(1+k-m) a_{k}^{\zeta}\left(F, P_{\varepsilon}, A\right) t^{(k-m) / 2} .
\end{aligned}
$$

Setting $k=n-1$ and equating terms in the asymptotic expansions establishes Assertion (1a). Similarly, we compute:

$$
\begin{aligned}
& \sum_{n} \partial_{\varepsilon} a_{n}^{\zeta}\left(1, P_{\varepsilon}, A\right) t^{(n-m) / 2} \sim \partial_{\varepsilon} \operatorname{Tr}\left\{e^{-t P_{\varepsilon, A}^{2}}\right\} \\
= & -2 t \operatorname{Tr}\left\{F P_{\varepsilon} e^{-t P_{\varepsilon, A}^{2}}\right\} \sim \sum_{k}-2 a_{k}^{\eta}\left(F, P_{\varepsilon}, A\right) t^{(k-m+1) / 2} .
\end{aligned}
$$

Again, equating coefficients in the associated asymptotic expansions yields Assertion (1b); the proof of Assertion (2) is similar and is therefore omitted. To prove Assertion (3), we compute:

$$
\begin{aligned}
& \sum_{n} \partial_{\varepsilon} a_{n}^{\eta}\left(1, P_{\varepsilon}, A\right) t^{(n-m-1) / 2} \sim \partial_{\varepsilon} \operatorname{Tr}\left\{P_{\varepsilon} e^{-t P_{\varepsilon, A}^{2}}\right\} \\
= & -\operatorname{Tr}\left\{f\left(P_{\varepsilon}-2 t P_{\varepsilon}^{3}\right) e^{-t P_{\varepsilon, A}^{2}}\right\}=-\left(1+2 t \partial_{t}\right) \operatorname{Tr}\left\{f P_{\varepsilon} e^{-t P_{\varepsilon, A}^{2}}\right\} \\
= & -\sum_{n}(1+(n-m-1)) a_{n}^{\eta}\left(f, P_{\varepsilon}, A\right) t^{(n-m-1) / 2} .
\end{aligned}
$$

Assertion (3) now follows by equating coefficients in the asymptotic expansions.

We will need the following Lemma to apply Lemma 2.4 It involves a formula for endomorphism valued smearing functions which is related to the product case and which generalizes the formula of Theorem 1.1 (3).

Lemma 2.5. Assume that the metric on $M$ is product near the boundary, that $P_{A}$ is self-adjoint, that $A$ is self-adjoint, and that the coefficients of $P$ and of $A$ are independent of the normal variable near the boundary. Let $F$ be an endomorphism of $V$ whose coefficients are independent of the normal variable near the boundary. If $m \geq 3$, then

$$
\begin{aligned}
a_{2}^{\zeta}(F, P, A) & =(4 \pi)^{-m / 2} \int_{M} \operatorname{Tr}\left\{F\left(\frac{1}{6} \tau+E\right)\right\} d x \\
& -\frac{1}{2(m-2)}(4 \pi)^{-m / 2} \int_{\partial M} \operatorname{Tr}\left\{(3-m) F \psi_{A}+F \gamma_{a}^{T} \psi_{A} \gamma_{a}^{T}\right\} d y
\end{aligned}
$$


Remark 2.6. To ensure that $P_{A}$ is self-adjoint, we impose the relations of Lemma 2.1 (3). Since $L_{a a}=0$ by assumption, this means that $\psi_{A}=\gamma_{m} \psi_{A} \gamma_{m}$ and hence $\operatorname{Tr}\left\{\psi_{A}\right\}=0$. Thus $a_{2}^{\zeta, \partial M}(\mathrm{Id}, P, A)=0$; this is in agreement with Theorem 1.1 (3).

Proof. We refer to 12 for the determination of the interior integrand. Let $N=\partial M$. We apply Theorem 1.1 to the operator $A$ on the closed manifold $N$ to see

$$
a_{2}^{\zeta}(1, A)=-\frac{1}{12}(4 \pi)^{-(m-1) / 2} \int_{N} \operatorname{Tr}\left\{\tau \operatorname{Id}+(12-6(m-1)) \psi_{A}^{2}+6 \psi_{A} \gamma_{a}^{T} \psi_{A} \gamma_{a}^{T}\right\} d y
$$

We set $A_{\varepsilon}:=A+\varepsilon F$. By Lemma 2.4 with an appropriate dimension shift,

$$
\begin{aligned}
& -2 a_{1}^{\eta}(F, A)=\left.\partial_{\varepsilon}\right|_{\varepsilon=0} a_{2}^{\zeta}\left(1, A_{\varepsilon}\right) \\
= & -\frac{1}{6}(4 \pi)^{-(m-1) / 2} \int_{N} \operatorname{Tr}\left\{F\left[(18-6 m) \psi_{A}+6 \gamma_{a}^{T} \psi_{A} \gamma_{a}^{T}\right]\right\} d y .
\end{aligned}
$$

Combining this result with Lemma 2.2(1) then yields:

$$
\begin{aligned}
& a_{2}^{\zeta, \partial M}(F, P, A)=-\frac{1}{2(m-2) \sqrt{\pi}} a_{1}^{\eta}(F, A) \\
= & -\frac{1}{12(m-2)}(4 \pi)^{-m / 2} \int_{\partial M} \operatorname{Tr}\left\{(18-6 m) F \psi_{A}+6 F \gamma_{a}^{T} \psi_{A} \gamma_{a}^{T}\right\} d y .
\end{aligned}
$$

\section{A Formula With universal COEFFiCiEnts}

As $a_{n}^{\eta}(F,-P, A)=-a_{n}^{\eta}(F, P, A)$, the boundary contributions, which are homogeneous of weight $n-1$, must be odd functions of $P$. Consequently, they vanish for $n=0,1$; Assertions (1) and (2) of Theorem 1.2 now follow. Furthermore, we have:

Lemma 3.1. There exist universal constants $c_{i}(m)$ so that

(1) $a_{2}^{\eta, \partial M}(f, P, A)=(4 \pi)^{-(m-1) / 2} \int_{\partial M} f \operatorname{Tr}\left\{c_{m}^{1} \psi_{P}+c_{m}^{2} \gamma_{m} \psi_{A}\right\} d y$.

(2) $a_{3}^{\eta, \partial M}(f, P, A)=(4 \pi)^{-m / 2} \int_{\partial M} \operatorname{Tr}\left\{c_{m}^{3} f \gamma_{m} \psi_{P}^{2}+c_{m}^{4} f \gamma_{m} \gamma_{a}^{T} \psi_{P} \gamma_{a}^{T} \psi_{P}\right.$

$+c_{m}^{5} f \gamma_{m} \psi_{A}^{2}+c_{m}^{6} f \psi_{P} \psi_{A}+c_{m}^{7} f \gamma_{m} \psi_{P} \gamma_{m} \psi_{A}+c_{m}^{8} f \gamma_{a}^{T} \psi_{P} \gamma_{a}^{T} \psi_{A}$

$+c_{m}^{9} f \gamma_{a} \psi_{P} \gamma_{a} \psi_{A}+c_{m}^{10} f \gamma_{m} \gamma_{a}^{T} \psi_{A} \gamma_{a}^{T} \psi_{A}+c_{m}^{11} f \psi_{P ; m}$

$+c_{m}^{12} f L_{a a} \psi_{P}+c_{m}^{13} f_{; m} \psi_{P}+c_{m}^{14} f\left(\gamma_{a}^{T} \psi_{P}\right)_{: a}+c_{m}^{15} f L_{a a} \gamma_{m} \psi_{A}$

$\left.+c_{m}^{16} f_{; m} \gamma_{m} \psi_{A}+c_{m}^{17} f\left(\gamma_{a} \psi_{A}\right)_{: a}\right\} d y$.

Many invariants do not occur because the trace over an odd number of $\gamma$-matrices is zero. Furthermore, invariants of the form $W_{a b} \gamma_{m} \gamma_{a} \gamma_{b}$ and $W_{a m} \gamma_{a}$ are omitted as their trace vanishes as well.

We begin our study of these coefficients by varying the compatible connection chosen:

Lemma 3.2. We have the relations:

(1) $c_{m}^{3}=0$.

(2) $c_{m}^{6}-c_{m}^{7}+(m-1) c_{m}^{8}+(m-1) c_{m}^{9}=0$.

(3) $c_{m}^{6}+c_{m}^{7}+(m-3) c_{m}^{8}-(m-3) c_{m}^{9}+2(m-3) c_{m}^{4}=0$.

(4) $c_{m}^{6}+c_{m}^{7}-(m-3) c_{m}^{8}+(m-3) c_{m}^{9}+2(m-3) c_{m}^{10}=0$.

Proof. There always exist Hermitian connections so $\nabla \gamma=0$, see for example 11. There are, however, many such connections. If $\omega:=\varrho_{i} e^{i}$ is a purely imaginary 1 form, then $\tilde{\nabla}:=\nabla-\omega$ Id is again a Hermitian connection with $\tilde{\nabla} \gamma=0$. One has

$$
\tilde{\psi}_{P}=\psi_{P}+\varrho_{i} \gamma_{i} \text { and } \tilde{\psi}_{A}=\psi_{A}+\varrho_{b} \gamma_{b}^{T} \text {. }
$$

Clearly $a_{n}^{\eta}$ does not depend on the particular connection chosen. We exhibit the terms which are linear in $\varrho$ and omit the remaining terms to derive the following 
equations from which the desired relations of the Lemma will follow:

$$
\begin{aligned}
& \operatorname{Tr}\left\{c_{m}^{3} \gamma_{m} \tilde{\psi}_{P}^{2}\right\}=-2 c_{m}^{3} \varrho_{m} \operatorname{Tr}\left\{\psi_{P}\right\}+\ldots, \\
& \operatorname{Tr}\left\{c_{m}^{4} \gamma_{m} \gamma_{a}^{T} \tilde{\psi}_{P} \gamma_{a}^{T} \tilde{\psi}_{P}\right\}=c_{m}^{4} \operatorname{Tr}\left\{-2(m-3) \gamma_{m} \varrho_{b} \gamma_{b} \psi_{P}\right\}+\ldots, \\
& \operatorname{Tr}\left\{c_{m}^{5} \gamma_{m} \tilde{\psi}_{A}^{2}\right\}=0+\ldots, \\
& \operatorname{Tr}\left\{c_{m}^{6} \tilde{\psi}_{P} \tilde{\psi}_{A}\right\}=c_{m}^{6} \operatorname{Tr}\left\{\varrho_{m} \gamma_{m} \psi_{A}+\varrho_{b} \gamma_{b} \psi_{A}+\psi_{P} \varrho_{b} \gamma_{b}^{T}\right\}+\ldots, \\
& \operatorname{Tr}\left\{c_{m}^{7} \gamma_{m} \tilde{\psi}_{P} \gamma_{m} \tilde{\psi}_{A}\right\}=c_{m}^{7} \operatorname{Tr}\left\{-\varrho_{m} \gamma_{m} \psi_{A}+\varrho_{b} \gamma_{b} \psi_{A}+\psi_{P} \varrho_{b} \gamma_{b}^{T}\right\}+\ldots, \\
& \operatorname{Tr}\left\{c_{m}^{8} \gamma_{a}^{T} \tilde{\psi}_{P} \gamma_{a}^{T} \tilde{\psi}_{A}\right\}=c_{m}^{8} \operatorname{Tr}\left\{(m-1) \varrho_{m} \gamma_{m} \psi_{A}-(m-3) \varrho_{b} \gamma_{b} \psi_{A}\right. \\
& \left.\quad \quad+(m-3) \psi_{P} \varrho_{b} \gamma_{b}^{T}\right\}+\ldots, \\
& \operatorname{Tr}\left\{c_{m}^{9} \gamma_{a} \tilde{\psi}_{P} \gamma_{a} \tilde{\psi}_{A}\right\}=c_{m}^{9} \operatorname{Tr}\left\{(m-1) \varrho_{m} \gamma_{m} \psi_{A}+(m-3) \varrho_{b} \gamma_{b} \psi_{A}\right. \\
& \left.\quad-(m-3) \psi_{P} \varrho_{b} \gamma_{b}^{T}\right\}+\ldots \\
& \operatorname{Tr}\left\{c_{m}^{10} \gamma_{m} \gamma_{a}^{T} \tilde{\psi}_{A} \gamma_{a}^{T} \tilde{\psi}_{A}\right\}=c_{m}^{10} \operatorname{Tr}\left\{2(m-3) \gamma_{m} \varrho_{b} \gamma_{b}^{T} \psi_{A}\right\}+\ldots
\end{aligned}
$$

We shift the spectrum of $A$ to show:

Lemma 3.3. We have the relations:

(1) $c_{m}^{5}=0$.

(2) $c_{m}^{6}=c_{m}^{7}$ and $c_{m}^{8}=-c_{m}^{9}$.

Proof. If we replace $A$ by $A+\varepsilon \mathrm{Id}$, then the boundary condition is unchanged for small values of $\varepsilon$. We set $\tilde{\psi}_{A}:=\psi_{A}+\varepsilon \mathrm{Id}$, exhibit only the linear terms, and omit all terms which are not linear in $\varepsilon$ to derive the following equations:

$$
\begin{aligned}
& \operatorname{Tr}\left\{c_{m}^{5} \gamma_{m} \tilde{\psi}_{A}^{2}\right\}=2 c_{m}^{5} \operatorname{Tr}\left\{\gamma_{m} \varepsilon \psi_{A}\right\}+\ldots, \\
& \operatorname{Tr}\left\{c_{m}^{6} \psi_{P} \tilde{\psi}_{A}\right\}=c_{m}^{6} \operatorname{Tr}\left\{\varepsilon \psi_{P}\right\}+\ldots, \\
& \operatorname{Tr}\left\{c_{m}^{7} \gamma_{m} \psi_{P} \gamma_{m} \tilde{\psi}_{A}\right\}=-c_{m}^{7} \operatorname{Tr}\left\{\varepsilon \psi_{P}\right\}+\ldots, \\
& \operatorname{Tr}\left\{c_{m}^{8} \gamma_{a}^{T} \psi_{P} \gamma_{a}^{T} \tilde{\psi}_{A}\right\}=c_{m}^{8} \operatorname{Tr}\left\{-(m-1) \varepsilon \psi_{P}\right\}+\ldots, \\
& \operatorname{Tr}\left\{c_{m}^{9} \gamma_{a} \psi_{P} \gamma_{a} \tilde{\psi}_{A}\right\}=c_{m}^{9} \operatorname{Tr}\left\{-(m-1) \varepsilon \psi_{P}\right\}+\ldots \\
& \operatorname{Tr}\left\{c_{m}^{10} \gamma_{m} \gamma_{a}^{T} \tilde{\psi}_{A} \gamma_{a}^{T} \tilde{\psi}_{A}\right\}=0+\ldots
\end{aligned}
$$

Assertion (1) follows. Furthermore, we have

$$
0=c_{m}^{6}-c_{m}^{7}-(m-1) c_{m}^{8}-(m-1) c_{m}^{9} .
$$

Assertion (2) follows from this equation and from Lemma 3.2 (2).

Lemma 3.4. We have $c_{m}^{14}=0$ and $c_{m}^{17}=0$.

Proof. We work on the flat annulus $M:=\mathbb{T}^{m-1} \times[0,1]$. Let $h_{a}$ and $H_{a}$ be real smooth functions on $M$. We set

$$
P=\gamma_{i} \partial_{i}^{x}+\varepsilon h_{a} \gamma_{a}^{T} \text { and } A=\gamma_{a}^{T} \partial_{a}^{y}+\varepsilon H_{b} \gamma_{b} .
$$

Let $F=f$. Id be scalar. The presence of the smearing function $f$ ensures the boundary and interior integrals do not interact. Modulo terms which are $O\left(\varepsilon^{2}\right)$, one has:

$$
a_{3}^{\eta}(F, P, A)=-\varepsilon(4 \pi)^{-m / 2} \int_{\partial M} \operatorname{Tr}\left(f\left\{c_{m}^{14} h_{b: b}+c_{m}^{17} H_{b: b}\right\}\right) d y+O\left(\varepsilon^{2}\right) .
$$

By Lemma 2.1

$$
\begin{array}{ll}
P^{*}=\gamma_{i} \partial_{i}^{x}-\varepsilon h_{a} \gamma_{a}^{T}, & \psi_{P^{*}}=-\varepsilon h_{a} \gamma_{a}^{T}, \\
A^{\#}=\gamma_{m} A^{*} \gamma_{m}=\gamma_{m}\left(\gamma_{a}^{T} \partial_{a}^{y}-\varepsilon H_{b} \gamma_{b}\right) \gamma_{m}, & \psi_{A^{\#}}=-\varepsilon H_{b} \gamma_{b} .
\end{array}
$$

Consequently, there is a sign change

$$
a_{3}^{\eta}\left(F, P^{*}, A^{\#}\right)=\varepsilon(4 \pi)^{-m / 2} \int_{\partial M} \operatorname{Tr}\left(f\left\{c_{m}^{14} h_{b: b}+c_{m}^{17} H_{b: b}\right\}\right) d y+O\left(\varepsilon^{2}\right) .
$$

By Lemma 2.3 $a_{3}^{\eta}\left(f \mathrm{Id}, P^{*}, A^{\#}\right)=a_{3}^{\eta}(f \mathrm{Id}, P, A)$; the Lemma follows. 
We use conformal variations to show:

Lemma 3.5. $c_{m}^{15}=\frac{m-3}{1-m} c_{m}^{16}$.

Proof. Let $f$ be a smooth function with $\left.f\right|_{\partial M}=0$. Let $d s^{2}(\varepsilon)=e^{2 \varepsilon f} d s^{2}$ and let $P(\varepsilon):=e^{-\varepsilon f} P$. Let $\nabla$ be a unitary connection with $\nabla \gamma=0$. Let $x=\left(x_{1}, \ldots, x_{m}\right)$ be a system of local coordinates on $M$. Expand $P=\gamma^{\nu} \nabla_{\partial_{\nu}}+\psi_{P}$ and use the metric to lower indices and define $\gamma_{\nu}$. Define a smooth 1 parameter family of connections

$$
\nabla(\varepsilon)_{\partial_{\mu}}:=\nabla_{\partial_{\mu}}+\frac{\varepsilon}{2}\left\{f_{; \nu} \gamma^{\nu} \gamma_{\mu}+f_{; \mu}\right\}
$$

Results of [16] show $\nabla(\varepsilon) \gamma(\varepsilon)=0$ and $\nabla(\varepsilon)$ is unitary. Furthermore,

$$
\psi_{P}(\varepsilon)=e^{-\varepsilon f}\left\{\psi_{P}-\frac{m-1}{2} \varepsilon f_{; i} \gamma_{i}\right\} \quad \text { and } \quad \psi_{A}(\varepsilon)=\psi_{A} .
$$

We suppose $\psi_{P}=0$. We study the term $\operatorname{Tr}\left\{f_{; m} \gamma_{m} \psi_{A}\right\}$ and compute:

$$
\begin{aligned}
&\left.\partial_{\varepsilon}\right|_{\varepsilon=0} \operatorname{Tr}\left\{c_{m}^{6} \psi_{P} \psi_{A}+c_{m}^{7} \gamma_{m} \psi_{P} \gamma_{m} \psi_{A}\right\} \\
&=-\frac{m-1}{2}\left(c_{m}^{6}-c_{m}^{7}\right) \operatorname{Tr}\left\{f_{; m} \gamma_{m} \psi_{A}\right\}=0, \\
&\left.\partial_{\varepsilon}\right|_{\varepsilon=0} \operatorname{Tr}\left\{c_{m}^{8} \gamma_{a}^{T} \psi_{P} \gamma_{a}^{T} \psi_{A}+c_{m}^{9} \gamma_{a} \psi_{P} \gamma_{a} \psi_{A}\right\} \\
&=-\frac{(m-1)^{2}}{2}\left\{c_{m}^{8}+c_{m}^{9}\right\} \operatorname{Tr}\left\{f_{; m} \gamma_{m} \psi_{A}\right\}=0, \\
&\left.\partial_{\varepsilon}\right|_{\varepsilon=0} L_{a a}=(1-m) f_{; m} .
\end{aligned}
$$

We concentrate on the term $\operatorname{Tr}\left\{f_{; m} \gamma_{m} \psi_{A}\right\}$ and compute

$$
\begin{aligned}
& \left.\partial_{\varepsilon}\right|_{\varepsilon=0} a_{3}^{\eta}(1, P(\varepsilon), A)=(4 \pi)^{-m / 2} \int_{\partial M} c_{m}^{15} \operatorname{Tr}\left\{(1-m) f_{; m} \gamma_{m} \psi_{A}\right\} d y \\
= & (m-3) a_{3}^{\eta}(f, P(\varepsilon), A)=(4 \pi)^{-m / 2} \int_{\partial M} c_{m}^{16} \operatorname{Tr}\left\{(m-3) f_{; m} \gamma_{m} \psi_{A}\right\} d y .
\end{aligned}
$$

The Lemma now follows.

We study a variation of the form $P_{\varepsilon}:=P+\varepsilon$ Id to establish

\section{Lemma 3.6.}

(1) $c_{m}^{1}=\frac{2-m}{4}(\beta(m)-1)$.

(2) $c_{m}^{12}=\frac{3-m}{3}\left(1-\frac{3}{4} \pi \beta(m)\right)$ and $c_{m}^{13}=\frac{(m-3)(m-1)}{2(m-2)}\left(1-\frac{1}{2} \pi \beta(m)\right)$.

Proof. Let $\psi_{P}$ be self-adjoint. Set $\psi_{A}:=\frac{1}{2} L_{a a} \mathrm{Id}$; then $A^{\#}=A^{*}=A$ and $P_{A}$ is self-adjoint. Let $P_{\varepsilon}:=P+\varepsilon \mathrm{Id}$. By Theorem 1.1 and Lemma 2.4

$$
\begin{aligned}
& \left.\partial_{\varepsilon}\right|_{\varepsilon=0} a_{2}^{\eta, \partial M}\left(f, P_{\varepsilon}, A\right)=(4 \pi)^{-(m-1) / 2} \int_{M} c_{m}^{1} f \operatorname{Tr}\{\operatorname{Id}\} d y \\
= & (2-m) a_{1}^{\zeta, \partial M}(f, P, A)=(4 \pi)^{-(m-1) / 2} \frac{2-m}{4}(\beta(m)-1) \int_{\partial M} f \operatorname{Tr}\{\operatorname{Id}\} d y
\end{aligned}
$$

Assertion (1) follows. To establish Assertion (2), we compute:

$$
\begin{aligned}
& \operatorname{Tr}\left\{c_{m}^{6} \psi_{P} \psi_{A}+c_{m}^{7} \gamma_{m} \psi_{P} \gamma_{m} \psi_{A}\right\} \\
& \quad=\frac{1}{2}\left(c_{m}^{6}-c_{m}^{7}\right) \operatorname{Tr}\left\{\psi_{P} L_{a a}\right\}=0, \\
& \operatorname{Tr}\left\{c_{m}^{8} \gamma_{a}^{T} \psi_{P} \gamma_{a}^{T} \psi_{A}+c_{m}^{9} \gamma_{a} \psi_{P} \gamma_{a} \psi_{A}\right\} \\
& \quad=\frac{(1-m)}{2}\left(c_{m}^{8}+c_{m}^{9}\right) \operatorname{Tr}\left\{\psi_{P} L_{a a}\right\}=0, \\
& \left.\partial_{\varepsilon}\right|_{\varepsilon=0} c_{m}^{4} \operatorname{Tr}\left\{\gamma_{m} \gamma_{a}^{T} \psi_{P} \gamma_{a}^{T} \psi_{P}\right) \\
& =c_{m}^{4} \operatorname{Tr}\left(\gamma_{m} \gamma_{a}^{T} \gamma_{a}^{T} \psi_{P}+\gamma_{a}^{T} \gamma_{m} \gamma_{a}^{T} \psi_{P}\right)=0 .
\end{aligned}
$$

Consequently again by Theorem 1.1 and Lemma 2.4 one has:

$$
\begin{aligned}
& \left.\partial_{\varepsilon}\right|_{\varepsilon=0} a_{3}^{\eta, \partial M}\left(f, P_{\varepsilon}, A\right)=(4 \pi)^{-m / 2} \int_{\partial M} \operatorname{Tr}\left\{c_{m}^{13} f_{; m} \operatorname{Id}+c_{m}^{12} f L_{a a}\right\} d y \\
= & (3-m) a_{2}^{\zeta, \partial M}(f, P, A) \\
= & (3-m) \int_{\partial M}\left\{\frac{1}{3}\left(1-\frac{3}{4} \pi \beta(m)\right) L_{a a} f-\frac{m-1}{2(m-2)}\left(1-\frac{1}{2} \pi \beta(m)\right) f_{; m}\right\} \operatorname{Tr}\{\operatorname{Id}\} d y .
\end{aligned}
$$

Assertion (2) follows. 


\section{The Variation $P_{\varepsilon}:=P+\varepsilon F$}

In this section, we will study $\partial_{\varepsilon} a_{3}^{\eta}\left(1, P_{\varepsilon}, A\right)$. There is a non-trivial interaction between the boundary and interior integrals that must be dealt with. Our basic identity is provided by Lemma 2.4

$$
\left.\partial_{\varepsilon}\right|_{\varepsilon=0} a_{3}^{\eta}\left(1, P_{\varepsilon}, A\right)=(3-m) a_{2}^{\zeta}(F, P, A) .
$$

Let $F$ be endomorphism valued. Then:

$$
\begin{aligned}
& \left.\partial_{\varepsilon}\right|_{\varepsilon=0} a_{3}^{\eta, M}\left(1, P_{\varepsilon}, A\right)=-\frac{1}{12}(4 \pi)^{-m / 2} \int_{M} \operatorname{Tr}\left\{\left[2(m-1) F_{; i}+3(4-m) F \gamma_{i} \psi_{P}\right.\right. \\
& \left.\quad+3(4-m) F \psi_{P} \gamma_{i}+3 F \gamma_{j} \gamma_{i} \psi_{P} \gamma_{j}+3 F \gamma_{j} \psi_{P} \gamma_{j} \gamma_{i}\right]_{; i} \\
& \quad+(3-m)\left[F \tau+6 F \gamma_{i} \gamma_{j} W_{i j}-6 F \psi_{P ; i} \gamma_{i}-6 F_{; i} \gamma_{i} \psi_{P}+3(4-m) F \psi_{P} \psi_{P}\right. \\
& \left.\left.\quad+3 F \psi_{P} \gamma_{i} \psi_{P} \gamma_{i}+3 F \gamma_{i} \psi_{P} \gamma_{i} \psi_{P}+3 F \gamma_{i} \psi_{P} \psi_{P} \gamma_{i}\right]\right\} d x .
\end{aligned}
$$

On the other hand, by Lemma 2.5

$$
\begin{aligned}
& a_{2}^{\zeta, M}(F, P, A)=-\frac{1}{12}(4 \pi)^{-m / 2} \int_{M} \operatorname{Tr}\left\{F \left(\tau+6 \gamma_{i} \gamma_{j} W_{i j}+6 \gamma_{i} \psi_{P ; i}-6 \psi_{P ; i} \gamma_{i}\right.\right. \\
& \left.\left.+12 \psi_{P}^{2}+3 \psi_{P} \gamma_{i} \psi_{P} \gamma_{i}+3 \gamma_{i} \psi_{P} \psi_{P} \gamma_{i}+3 \gamma_{i} \psi_{P} \gamma_{i} \psi_{P}-3 m \psi_{P}^{2}\right)\right\} d x
\end{aligned}
$$

Consequently, we may integrate by parts to see

$$
\begin{aligned}
& \left.\partial_{\varepsilon} a_{3}^{\eta, M}\left(1, P_{\varepsilon}, A\right)\right|_{\varepsilon=0}-(3-m) a_{2}^{\zeta, M}(F, P, A) \\
= & -\frac{1}{12}(4 \pi)^{-m / 2} \int_{M} \operatorname{Tr}\left\{\left[2(m-1) F_{; i}+3(4-m) F \gamma_{i} \psi_{P}+3(4-m) F \psi_{P} \gamma_{i}\right.\right. \\
& \left.\left.+3 F \gamma_{j} \gamma_{i} \psi_{P} \gamma_{j}+3 \gamma_{j} \psi_{P} \gamma_{j} \gamma_{i} F\right]_{; i}-6(3-m) F_{; i} \gamma_{i} \psi_{P}-6(3-m) F \gamma_{i} \psi_{P ; i}\right\} d x \\
= & \frac{1}{12}(4 \pi)^{-m / 2} \int_{\partial M} \operatorname{Tr}\left\{2(m-1) F_{; m}+3(4-m) F \gamma_{m} \psi_{P}+3(4-m) F \psi_{P} \gamma_{m}\right. \\
& \left.+3 F \gamma_{j} \gamma_{m} \psi_{P} \gamma_{j}+3 F \gamma_{j} \psi_{P} \gamma_{j} \gamma_{m}-6(3-m) F \gamma_{m} \psi_{P}\right\} d y .
\end{aligned}
$$

After setting $c_{m}^{3}=c_{m}^{5}=0, c_{m}^{7}=c_{m}^{6}$, and $c_{m}^{9}=-c_{m}^{8}$, one has

$$
\begin{aligned}
& \left.\partial_{\varepsilon} a_{3}^{\eta, \partial M}\left(1, P_{\varepsilon}, A\right)\right|_{\varepsilon=0}=(4 \pi)^{-m / 2} \int_{\partial M} \operatorname{Tr}\left\{c_{m}^{4} F\left(\gamma_{a}^{T} \psi_{P} \gamma_{m} \gamma_{a}^{T}+\gamma_{m} \gamma_{a}^{T} \psi_{P} \gamma_{a}^{T}\right)\right. \\
& \quad+c_{m}^{6} F\left(\psi_{A}+\gamma_{m} \psi_{A} \gamma_{m}\right)+c_{m}^{8} F\left(\gamma_{a}^{T} \psi_{A} \gamma_{a}^{T}-\gamma_{a} \psi_{A} \gamma_{a}\right)+c_{m}^{11} F_{; m} \\
& \left.\quad+c_{m}^{12} F L_{a a}\right\} d y
\end{aligned}
$$

There are several different settings where we know $a_{2}^{\zeta, \partial M}$. For the next two lemmas, to ensure that $P_{A}$ is self adjoint, we shall assume $\psi_{P}$ and $\psi_{A}$ are self adjoint and that $\psi_{A}=\gamma_{m} \psi_{A} \gamma_{m}+L_{a a}$ Id. We begin by applying Theorem 1.1]

Lemma 4.1. We have $c_{m}^{11}=\frac{(m-3)(m-1)}{2(m-2)}\left(1-\frac{1}{2} \pi \beta(m)\right)-\frac{1}{6}(m-1)$.

Proof. We take $F=f$. Id to be scalar and set $P_{\varepsilon}:=P+\varepsilon F$. The terms involving $\operatorname{Tr}\left(\psi_{A}\right)$ and $\operatorname{Tr}\left(\gamma_{m} \psi_{P}\right)$ cancel and we have

$$
\begin{aligned}
0 & =\left.\partial_{\varepsilon} a_{3}^{\eta}\left(1, P_{\varepsilon}, A\right)\right|_{\varepsilon=0}-(3-m) a_{2}^{\zeta}(F, P, A) \\
& =\operatorname{Tr}\{\operatorname{Id}\}(4 \pi)^{-m / 2} \int_{\partial M}\left\{\frac{1}{6}(m-1)+c_{m}^{11}\right) f_{; m}+c_{m}^{12} f L_{a a} \\
& \left.-\frac{3-m}{3}\left(1-\frac{3}{4} \pi \beta(m)\right) L_{a a} f+\frac{(m-1)(3-m)}{2(m-2)}\left(1-\frac{1}{2} \pi \beta(m)\right) f_{; m}\right\} d y
\end{aligned}
$$

We equate the coefficients of $f L_{a a}$ to determine a value for $c_{m}^{12}$ which agrees with that obtained in Lemma 3.6. Equating the coefficients of $f_{; m}$ determines $c_{m}^{11}$.

We apply Lemma 2.5 to prove:

Lemma 4.2. We have the relations:

(1) $c_{m}^{6}=-\frac{(3-m)^{2}}{4(m-2)}$, and $c_{m}^{8}=-\frac{3-m}{4(m-2)}$.

(2) $c_{m}^{4}=0$, and $c_{m}^{10}=-2 \frac{(3-m)}{4(m-2)}$. 
Proof. We assume the structures are product near the boundary. We first study the terms $\operatorname{Tr}\left\{F \psi_{A}\right\}$ and $\operatorname{Tr}\left\{\gamma_{a} F \gamma_{a} \psi_{A}\right\}$. Since $L_{a a}=0, \gamma_{m} \psi_{A} \gamma_{m}=\psi_{A}$. We compute using equations (4.b) and (4.C) that

$$
\begin{aligned}
& \left.c_{m}^{6} \partial_{\varepsilon}\right|_{\varepsilon=0} \operatorname{Tr}\left\{\psi_{P} \psi_{A}+\gamma_{m} \psi_{P} \gamma_{m} \psi_{A}\right\}=2 c_{m}^{6} \operatorname{Tr}\left\{F \psi_{A}\right\}, \\
& \left.c_{m}^{8} \partial_{\varepsilon}\right|_{\varepsilon=0} \operatorname{Tr}\left\{\gamma_{a}^{T} \psi_{P} \gamma_{a}^{T} \psi_{A}-\gamma_{a} \psi_{P} \gamma_{a} \psi_{A}\right\}=-2 c_{m}^{8} \operatorname{Tr}\left\{\gamma_{a} F \gamma_{a} \psi_{A}\right\} .
\end{aligned}
$$

Thus Lemma 2.5 yields:

$$
\begin{aligned}
& (4 \pi)^{-m / 2} \int_{\partial M} \operatorname{Tr}\left\{2 c_{m}^{6} F \psi_{A}-2 c_{m}^{8} F \gamma_{a} \psi_{A} \gamma_{a}\right\} d y+\ldots \\
= & -\frac{3-m}{2(m-2)}(4 \pi)^{-m / 2} \int_{\partial M} \operatorname{Tr}\left\{F(3-m) \psi_{A}-F \gamma_{a} \psi_{A} \gamma_{a}\right\} d y+\ldots
\end{aligned}
$$

To complete the proof of Assertion (1), we must show Equation 4.d yields two linearly independent relations. If we set $F=\psi_{A}=\sqrt{-1} \gamma_{1}$, then $\psi_{A}^{*}=\psi_{A}$, $\gamma_{m} \psi_{A} \gamma_{m}=\psi_{A}$, and

$$
\operatorname{Tr}\left(F \psi_{A}\right)=\operatorname{Tr}\{\operatorname{Id}\} \quad \text { and } \quad \operatorname{Tr}\left(F \gamma_{a} \psi_{A} \gamma_{a}\right)=(m-3) \operatorname{Tr}\{\operatorname{Id}\} .
$$

If we set $F=\psi_{A}=\gamma_{1} \gamma_{2} \gamma_{3}$, then $\psi_{A}^{*}=\psi_{A}, \gamma_{m} \psi_{A} \gamma_{m}=A$, and

$$
\operatorname{Tr}\left(F \psi_{A}\right)=\operatorname{Tr}\{\operatorname{Id}\} \quad \text { and } \operatorname{Tr}\left(F \gamma_{a} \psi_{a} \gamma_{a}\right)=(m-7) \operatorname{Tr}\{\operatorname{Id}\} .
$$

Assertion (1) follows. Assertion (1) and Lemma 3.2 imply Assertion (2).

\section{A special case calculation on the ball}

In order to find the remaining unknown coefficients $c_{m}^{2}$ and $c_{m}^{16}$ we evaluate the leading coefficients in the asymptotic of the eta invariant for an example on the ball. We first describe the setting considered.

Let $r \in[0,1]$ be the radial normal coordinate and $d \Sigma^{2}$ the usual metric on the unit sphere $S^{m-1}$. Then the standard metric on the ball is $d s^{2}=d r^{2}+r^{2} d \Sigma^{2}$. The inward unit normal on the boundary is $-\partial_{r}$. For this metric, the only nonvanishing components of the Christoffel symbols are

$$
\Gamma_{a b c}=\frac{1}{r} \tilde{\Gamma}_{a b c} \text { and } \Gamma_{a b m}=\frac{1}{r} \delta_{a b} ;
$$

the second fundamental form is given by $L_{a b}=\delta_{a b}$. We will use $\tilde{\Gamma}_{a b c}$ to refer to the Christoffel symbols associated with the metric $d \Sigma^{2}$ on the sphere $S^{m-1}$. We will consider the Dirac operator $P=\gamma^{\nu} \partial_{\nu}$ on the ball; we take the flat connection $\nabla$ and set $\psi_{P}=0$. We suppose $m$ even (there is a corresponding decomposition for $m$ odd) and use the following representation of the $\gamma$-matrices,

$$
\begin{aligned}
& \gamma_{a(m)}=\left(\begin{array}{cc}
0 & \sqrt{-1} \cdot \gamma_{a(m-1)} \\
-\sqrt{-1} \cdot \gamma_{a(m-1)} & 0
\end{array}\right) \text { and } \\
& \gamma_{m(m)}=\left(\begin{array}{cc}
0 & \sqrt{-1} \cdot 1_{m-1} \\
\sqrt{-1} \cdot 1_{m-1} & 0
\end{array}\right) .
\end{aligned}
$$

We stress that the matrices $\gamma_{j(m)}$ are the $\gamma$-matrices projected along some vielbein system $e_{j}$. We decompose $\nabla_{j}=e_{j}+\omega_{j}$ where $\omega_{j}=\frac{1}{4} \Gamma_{j k l} \gamma_{k(m)} \gamma_{l(m)}$ is the connection- 1 form of the spin connection. If $\tilde{\nabla}$ denotes the connection on the sphere, we have that

$$
\nabla_{a}=\frac{1}{r}\left(\left(\begin{array}{cc}
\tilde{\nabla}_{a} & 0 \\
0 & \tilde{\nabla}_{a}
\end{array}\right)+\frac{1}{2} \gamma_{a(m)}^{T}\right) .
$$

This allows us to decompose the Dirac operator on the ball into a radial part and a part living on the sphere. In detail, if $\tilde{P}$ is the Dirac operator on the sphere, we have

$$
P=\left(\frac{\partial}{\partial x_{m}}-\frac{m-1}{2 r}\right) \gamma_{m(m)}+\frac{1}{r}\left(\begin{array}{cc}
0 & \sqrt{-1} \tilde{P} \\
-\sqrt{-1} \tilde{P} & 0
\end{array}\right)
$$


Let $d_{s}$ be the dimension of the spin bundle on the disk; $d_{s}=2^{m / 2}$ if $m$ is even. The spinor modes $\mathcal{Z}_{ \pm}^{(n)}$ on the sphere are discussed in [13]. We have

$$
\begin{aligned}
& \tilde{P} \mathcal{Z}_{ \pm}^{(n)}(\Omega)= \pm\left(n+\frac{m-1}{2}\right) \mathcal{Z}_{ \pm}^{(n)}(\Omega) \text { for } n=0,1, \ldots ; \\
& d_{n}(m):=\operatorname{dim} \mathcal{Z}_{ \pm}^{(n)}(\Omega)=\frac{1}{2} d_{s}\left(\begin{array}{c}
m+n-2 \\
n
\end{array}\right) .
\end{aligned}
$$

Let $J_{\nu}(z)$ be the Bessel functions. These satisfy the differential equation [24]:

$$
\frac{d^{2} J_{\nu}(z)}{d z^{2}}+\frac{1}{z} \frac{d J_{\nu}(z)}{d z}+\left(1-\frac{\nu^{2}}{z^{2}}\right) J_{\nu}(z)=0 .
$$

Let $P \varphi_{ \pm}= \pm \mu \varphi_{ \pm}$be an eigen function of $P$. Modulo a suitable radial normalizing constant $C$, we may express:

$$
\begin{aligned}
\varphi_{ \pm}^{(+)} & =\frac{C}{r^{(m-2) / 2}}\left(\begin{array}{c}
\sqrt{-1} J_{n+m / 2}(\mu r) Z_{+}^{(n)}(\Omega) \\
\pm J_{n+m / 2-1}(\mu r) Z_{+}^{(n)}(\Omega)
\end{array}\right), \text { and } \\
\varphi_{ \pm}^{(-)} & =\frac{C}{r^{(m-2) / 2}}\left(\begin{array}{c} 
\pm J_{n+m / 2-1}(\mu r) Z_{-}^{(n)}(\Omega) \\
\sqrt{-1} J_{n+m / 2}(\mu r) Z_{-}^{(n)}(\Omega)
\end{array}\right) .
\end{aligned}
$$

We next impose the boundary conditions. We choose for $\epsilon \in \mathbb{R}$ the boundary endomorphism

$$
\psi_{A}=\epsilon \gamma_{m(m)}+\frac{1}{2} L_{a a} \mathrm{Id}
$$

such that

$$
\psi_{A}=\gamma_{m(m)} \psi_{A}^{*} \gamma_{m(m)}+L_{a a} \text { Id }
$$

This guarantees that $P_{A}$ is self-adjoint, see Lemma 2.1 Assertions (2) and (3). For this setting the general form of the leading coefficients for the eta invariant are obtained from Lemma 3.1 and Lemma 3.5 Noting that the volume of the $m-1$ dimensional sphere is $2 \pi^{m / 2} / \Gamma(m / 2)$, and that

$$
\operatorname{Tr}\left\{\gamma_{m} \psi_{A}\right\}=-\epsilon d_{s}
$$

one finds

$$
\begin{aligned}
& a_{2}^{\eta}(1, P, A)=-c_{m}^{2} \frac{\epsilon d_{s} \sqrt{\pi}}{2^{m-2} \Gamma\left(\frac{m}{2}\right)}, \\
& a_{3}^{\eta}(1, P, A)=c_{m}^{16} \frac{(m-3) \epsilon d_{s}}{2^{m-1} \Gamma\left(\frac{m}{2}\right)} .
\end{aligned}
$$

Thus finding explicit answers for this example will allow us to determine $c_{m}^{2}$ and $c_{m}^{16}$. We proceed towards this goal.

For the $\psi_{A}$ given in 5.C the boundary operator $A$ is given by

$$
A=\left(\begin{array}{cc}
-\tilde{P} & i \epsilon \\
i \epsilon & \tilde{P}
\end{array}\right) .
$$

We need to find the spectral projection on those eigen spinors of $A$ whose eigenvalues have a positive real part. The endomorphism $\psi_{A}$ chosen allows us to obtain closed forms for all eigenvalues $\pm \mu_{n}$ and eigenspinors $\left(\alpha_{1}^{ \pm}, \alpha_{2}^{ \pm}\right)$defined by the differential equation

$$
A\left(\begin{array}{c}
\alpha_{1}^{ \pm} \\
\alpha_{2}^{ \pm}
\end{array}\right)=\left(\begin{array}{cc}
-\tilde{P} & i \epsilon \\
i \epsilon & \tilde{P}
\end{array}\right)\left(\begin{array}{c}
\alpha_{1}^{ \pm} \\
\alpha_{2}^{ \pm}
\end{array}\right)= \pm \mu_{n}\left(\begin{array}{c}
\alpha_{1}^{ \pm} \\
\alpha_{2}^{ \pm}
\end{array}\right)
$$

Let

$$
\lambda_{n}=n+\frac{1}{2}(m-1)
$$


be the eigenvalues associated with $\epsilon=0$ 23. One can then show that

$$
\mu_{n}=\sqrt{\lambda_{n}^{2}-\epsilon^{2}}
$$

and

$$
\begin{aligned}
& \left(\begin{array}{c}
\alpha_{1}^{+} \\
\alpha_{2}^{+}
\end{array}\right)=\left(\begin{array}{c}
\frac{\sqrt{-1} \epsilon}{2 \lambda_{n}} Z_{+}^{(n)}+Z_{-}^{(n)} \\
\frac{1}{2 \lambda_{n}}\left(\sqrt{\lambda_{n}^{2}-\epsilon^{2}}+\lambda_{n}\right)^{(n)}+\frac{1}{\sqrt{-1} \epsilon}\left(\sqrt{\lambda_{n}^{2}-\epsilon^{2}}-\lambda_{n}\right) Z_{-}^{(n)}
\end{array}\right), \\
& \left(\begin{array}{c}
\alpha_{1}^{-} \\
\alpha_{2}^{-}
\end{array}\right)=\left(\begin{array}{c}
Z_{+}^{(n)}-\frac{\sqrt{-1} \epsilon}{2 \lambda_{n}} Z_{-}^{(n)} \\
-\frac{1}{\sqrt{-1} \epsilon}\left(\sqrt{\lambda_{n}^{2}-\epsilon^{2}}-\lambda_{n}\right) Z_{+}^{(n)}+\frac{1}{2 \lambda_{n}}\left(\sqrt{\lambda_{n}^{2}-\epsilon^{2}}+\lambda_{n}\right) Z_{-}^{(n)}
\end{array}\right) .
\end{aligned}
$$

We choose $\epsilon<(m-1) / 2$ such that all eigenvalues $\mu_{n}$ are real. The solutions are normalized such that in the limit $\epsilon \rightarrow 0$ they reduce to the previously determined solutions in 23 .

We want to suppress the projection on the positive spectrum of $A$. Using the solutions given in Equations (5.a) and (5.b) this is easily accomplished. Projecting $\varphi_{ \pm}^{(+)}$onto the positive spectrum of $A$ gives the implicit eigenvalue condition

$$
J_{\lambda_{n}-\frac{1}{2}}(\mu) \mp \frac{\epsilon}{\sqrt{\lambda_{n}^{2}-\epsilon^{2}}+\lambda_{n}} J_{\lambda_{n}+\frac{1}{2}}(\mu)=0,
$$

whereas projecting $\varphi_{ \pm}^{(-)}$produces

$$
J_{\lambda_{n}-\frac{1}{2}}(\mu) \pm \frac{1}{\epsilon}\left(\sqrt{\lambda_{n}^{2}-\epsilon^{2}}-\lambda_{n}\right) J_{\lambda_{n}+\frac{1}{2}}(\mu)=0
$$

Combining the equations for the positive eigenvalues of $P_{A}$, we have the condition

$$
\begin{aligned}
\left(J_{\lambda_{n}-\frac{1}{2}}(\mu)-\frac{\epsilon}{\sqrt{\lambda_{n}^{2}-\epsilon^{2}}+\lambda_{n}} J_{\lambda_{n}+\frac{1}{2}}(\mu)\right) \times \\
\left(J_{\lambda_{n}-\frac{1}{2}}(\mu)+\frac{1}{\epsilon}\left(\sqrt{\lambda_{n}^{2}-\epsilon^{2}}-\lambda_{n}\right) J_{\lambda_{n}+\frac{1}{2}}(\mu)\right)=0 .
\end{aligned}
$$

For the present purpose it will be sufficient to find the unknown multipliers $c_{m}^{2}$ and $c_{m}^{16}$ multiplying a linear term in $\psi_{A}$. Therefor we only need to pick up linear terms in $\epsilon$ and we will consider only terms up to the order $\epsilon$ explicitly. Having that in mind we write the implicit eigenvalue condition for positive eigenvalues instead as

$$
J_{\lambda_{n}-\frac{1}{2}}(\mu)\left(J_{\lambda_{n}-\frac{1}{2}}(\mu)-\frac{\epsilon}{\lambda_{n}} J_{\lambda_{n}+\frac{1}{2}}(\mu)\right)+\mathcal{O}\left(\epsilon^{2}\right)=0 .
$$

To simplify the notation, set

$$
p=\lambda_{n}-\frac{1}{2} \quad \text { and } \quad d_{n}(m)=d_{p}(m) .
$$

Furthermore, we use the recursion for Bessel functions, see 24],

$$
z \frac{d}{d z} J_{p}(z)-p J_{p}(z)=-z J_{p+1}(z)
$$

to rewrite (5.f) such that only the index $p$ appears,

$$
J_{p}(\mu)\left(J_{p}(\mu)\left[1-\frac{\epsilon p}{\mu(p+1 / 2)}\right]+\frac{\epsilon}{p+1 / 2} J_{p}^{\prime}(\mu)\right)+\mathcal{O}\left(\epsilon^{2}\right)=0 .
$$

Proceeding similarly with the negative eigenvalues of $P_{A}$ the outcome is

$$
J_{p}(\mu)\left(J_{p}(\mu)\left[1+\frac{\epsilon p}{\mu(p+1 / 2)}\right]-\frac{\epsilon}{p+1 / 2} J_{p}^{\prime}(\mu)\right)+\mathcal{O}\left(\epsilon^{2}\right)=0 .
$$

Using Cauchy's residue theorem these equations allow us to rewrite the eta function

$$
\eta(s ; P, A)=\sum_{\mu}(\operatorname{sign}(\mu))|\mu|^{-s}
$$


in terms of a contour integral, a technique recently described in detail in $8,9,10,29$. The coefficients in the asymptotic expansion (1.c) are then determined by evaluating residues of $\eta$ according to 21]

$$
\text { Res } \eta(m-n ; P, A)=\frac{2 a_{n}^{\eta}(1, P, A)}{\Gamma\left(\frac{m-n+1}{2}\right)} \text {. }
$$

We will need the residues at $s=m-2$ and $s=m-3$ in order to determine the coefficients $a_{2}^{\eta}$ and $a_{3}^{\eta}$.

Neglecting systematically the higher order terms in $\epsilon$, we use a suitable counterclockwise contour $C$ enclosing all the solutions of the Equations (5.g) and (5.h) to write the eta function as (from now on it will be understood that this is the eta function up to the order $\epsilon$ )

$$
\begin{aligned}
& \eta(s ; P, A)=\sum_{p} d_{p}(m) \int_{C} \frac{d k}{2 \pi i} k^{-s} \frac{\partial}{\partial k} \\
& \left\{\ln \left[J_{p}(k)\left(J_{p}(k)\left[1-\frac{\epsilon p}{k(p+1 / 2)}\right]+\frac{\epsilon}{p+1 / 2} J_{p}^{\prime}(k)\right)\right]\right. \\
& \left.-\ln \left[J_{p}(k)\left(J_{p}(k)\left[1+\frac{\epsilon p}{k(p+1 / 2)}\right]-\frac{\epsilon}{p+1 / 2} J_{p}^{\prime}(k)\right)\right]\right\} \\
& =\sum_{p} d_{p}(m) \int_{C} \frac{d k}{2 \pi i} k^{-s} \frac{\partial}{\partial k} \\
& \left\{\ln \left[1-\frac{\epsilon p}{k(p+1 / 2)}+\frac{\epsilon}{p+1 / 2} \frac{J_{p}^{\prime}(k)}{J_{p}(k)}\right]\right. \\
& \left.-\ln \left[1+\frac{\epsilon p}{k(p+1 / 2)}-\frac{\epsilon}{p+1 / 2} \frac{J_{p}^{\prime}(k)}{J_{p}(k)}\right]\right\} \\
& =\sum_{p} d_{p}(m) p^{-s} \int_{C} \frac{d z}{2 \pi i} z^{-s} \frac{\partial}{\partial z} \\
& \left\{\ln \left[1-\frac{\epsilon p}{z p(p+1 / 2)}+\frac{\epsilon}{p+1 / 2} \frac{J_{p}^{\prime}(z p)}{J_{p}(z p)}\right]\right. \\
& \left.-\ln \left[1+\frac{\epsilon p}{z p(p+1 / 2)}-\frac{\epsilon}{p+1 / 2} \frac{J_{p}^{\prime}(z p)}{J_{p}(z p)}\right]\right\} .
\end{aligned}
$$

In the last equation we substituted $k=z p$ in order to allow later on for a straightforward application of the formulas for the uniform asymptotic expansion of the Bessel functions. Again, expanding up to the order $\epsilon$ term, we write instead

$$
\begin{aligned}
\eta(s ; P, A)= & 2 \epsilon \sum_{p} d_{p}(m) p^{-s} \int_{C} \frac{d z}{2 \pi i} z^{-s} \frac{\partial}{\partial z} \\
& \left\{-\frac{1}{z(p+1 / 2)}+\frac{1}{p+1 / 2} \frac{J_{p}^{\prime}(z p)}{J_{p}(z p)}\right\} .
\end{aligned}
$$

The next step in the procedure is to shift the contour towards the imaginary axis, turning the Bessel function $J_{p}$ into the Bessel function $I_{p}$. In detail, we find

$$
\begin{aligned}
\eta(s ; P, A)= & -\frac{2 \epsilon}{\pi} \cos \left(\frac{\pi s}{2}\right) \sum_{p} d_{p}(m) p^{-s}(p+1 / 2)^{-1} \\
& \int_{0}^{\infty} d z z^{-s} \frac{d}{d z}\left\{\frac{1}{z}-\frac{I_{p}^{\prime}(z p)}{I_{p}(z p)}\right\} .
\end{aligned}
$$

The residues of the eta function are completely determined by the asymptotic behaviour of the Bessel functions, see [29] for details. Therefor we need to introduce 
some additional notation dealing with the uniform asymptotic expansion of the Bessel function $I_{p}(k)$. For $p \rightarrow \infty$ with $z=k / p$ fixed, we make use of the uniform asymptotic expansion of the Bessel function $I_{p}(z p)$ and the derivative $I_{p}^{\prime}(z p)$. In detail, the relevant results are [1,

$$
\begin{aligned}
& I_{p}(z p) \sim \frac{1}{\sqrt{2 \pi p}} \frac{e^{p \eta}}{\left(1+z^{2}\right)^{1 / 4}}\left[1+\sum_{l=1}^{\infty} \frac{u_{l}(t)}{p^{l}}\right], \\
& I_{p}^{\prime}(z p) \sim \frac{1}{\sqrt{2 \pi p}} \frac{e^{p \eta}\left(1+z^{2}\right)^{1 / 4}}{z}\left[1+\sum_{l=1}^{\infty} \frac{v_{l}(t)}{p^{l}}\right],
\end{aligned}
$$

where

$$
t=1 / \sqrt{1+z^{2}} \text { and } \eta=\sqrt{1+z^{2}}+\ln \left[z /\left(1+\sqrt{1+z^{2}}\right)\right] .
$$

Let $u_{0}(t)=1$. We use the recursion relationship given in 1 to determine the polynomials $u_{l}(t)$ and $v_{l}(t)$ which appear in Equations [5.jp and (5.k),

$$
\begin{aligned}
u_{l+1}(t) & =\frac{1}{2} t^{2}\left(1-t^{2}\right) u_{l}^{\prime}(t)+\frac{1}{8} \int_{0}^{t} d \tau\left(1-5 \tau^{2}\right) u_{l}(\tau), \\
v_{l}(t) & =u_{l}(t)+t\left(t^{2}-1\right)\left[\frac{1}{2} u_{l-1}(t)+t u_{l-1}^{\prime}(t)\right] .
\end{aligned}
$$

In particular we have

$$
u_{1}(t)=\frac{1}{8} t-\frac{5}{24} t^{3}, \quad v_{1}(t)=-\frac{3}{8} t+\frac{7}{24} t^{3} .
$$

The needed leading two contributions from the asymptotic expansion are then given by

$$
\begin{aligned}
B_{0}(s ; P, A)= & -\frac{2 \epsilon}{\pi} \cos \left(\frac{\pi s}{2}\right) \sum_{p} d_{p}(m) p^{-s}(p+1 / 2)^{-1} \\
& \int_{0}^{\infty} d z z^{-s} \frac{d}{d z}\left\{\frac{1}{z}\left(1-\sqrt{1+z^{2}}\right)\right\}, \\
B_{-1}(s ; P, A)= & \frac{2 \epsilon}{\pi} \cos \left(\frac{\pi s}{2}\right) \sum_{p} d_{p}(m) p^{-s}(p+1 / 2)^{-1} \\
& \int_{0}^{\infty} d z z^{-s} \frac{d}{d z}\left\{\frac{\sqrt{1+z^{2}}}{z}\left(\frac{1}{p}\left[v_{1}(t)-u_{1}(t)\right]\right)\right\} \\
= & -\frac{\epsilon}{\pi} \cos \left(\frac{\pi s}{2}\right) \sum_{p} d_{p}(m) p^{-s-1}(p+1 / 2)^{-1} \\
& \int_{0}^{\infty} d z z^{-s} \frac{d}{d z} \frac{z}{1+z^{2}} .
\end{aligned}
$$

The integrals can be evaluated with the help of the beta function, see 24]. Using

$$
\Gamma\left(-\frac{1+s}{2}\right)=-\frac{\pi}{\cos \left(\frac{\pi s}{2}\right) \Gamma\left(\frac{3+s}{2}\right)}
$$


the answers obtained are

$$
\begin{aligned}
B_{0}\left(s ; P_{A}\right) & =-\frac{\epsilon}{\pi} \cos \left(\frac{\pi s}{2}\right) \frac{\Gamma\left(-\frac{s+1}{2}\right) \Gamma\left(1+\frac{s}{2}\right)}{\sqrt{\pi}} \sum_{p} d_{p}(m) p^{-s}(p+1 / 2)^{-1} \\
& =\epsilon \frac{\Gamma\left(1+\frac{s}{2}\right)}{\sqrt{\pi} \Gamma\left(\frac{3+s}{2}\right)} \sum_{p} d_{p}(m) p^{-s}(p+1 / 2)^{-1} \\
B_{-1}\left(s ; P_{A}\right) & =-\frac{s \epsilon}{2} \sum_{p} d_{p}(m) p^{-s-1}(p+1 / 2)^{-1}
\end{aligned}
$$

The remaining summations are related to the spectrum on the sphere. Let $d:=$ $m-1$. We define the base zeta-function $\zeta_{S^{d}}$ and the Barnes zeta-function $\zeta_{\mathcal{B}}$ [6],

$$
\zeta_{S^{d}}(s)=\sum_{n=0}^{\infty} d_{n}(m) p^{-2 s} \text { and } \zeta_{\mathcal{B}}(s, a)=\sum_{n=0}^{\infty} d_{n}(m)(n+a)^{-s}
$$

We then have the relation

$$
\zeta_{S^{d}}(s)=\frac{1}{2} d_{s} \zeta_{\mathcal{B}}\left(2 s, \frac{m}{2}-1\right)
$$

Using the Barnes zeta-function, up to terms that are irrelevant for the present purpose because their residues are located to the left of $s=m-3$, we find

$$
\begin{aligned}
B_{0}(s ; P, A) & =\frac{1}{2} d_{s} \epsilon \frac{\Gamma\left(1+\frac{s}{2}\right)}{\sqrt{\pi} \Gamma\left(\frac{3+s}{2}\right)}\left\{\zeta_{\mathcal{B}}\left(s+1, \frac{m}{2}-1\right)-\frac{1}{2} \zeta_{\mathcal{B}}\left(s+2, \frac{m}{2}-1\right)+\ldots\right\}, \\
B_{-1}(s ; P, A) & =-\frac{1}{4} s \epsilon d_{s}\left\{\zeta_{\mathcal{B}}\left(s+2, \frac{m}{2}-1\right)+\ldots\right\} .
\end{aligned}
$$

This reduces the analysis of the eta function on the ball to the analysis of $\zeta_{\mathcal{B}}(s, a)$. To compute the relevant residues, we first express $\zeta_{\mathcal{B}}(s, a)$ as a contour integral. Let $\mathcal{C}$ be the Hankel contour.

$$
\begin{aligned}
\zeta_{\mathcal{B}}(s, a) & =\sum_{n=0}^{\infty}\left(\begin{array}{c}
d+n-1 \\
n
\end{array}\right)(n+a)^{-s}=\sum_{\vec{m} \in \mathbb{N}_{0}^{d}}\left(a+m_{1}+\ldots+m_{d}\right)^{-s} \\
& =\frac{\Gamma(1-s)}{2 \pi} \int_{\mathcal{C}} d t(-t)^{s-1} \frac{e^{-a t}}{\left(1-e^{-t}\right)^{d}} .
\end{aligned}
$$

The residues of $\zeta_{\mathcal{B}}(s, a)$ are intimately connected with the generalized Bernoulli polynomials 38,

$$
\frac{e^{-a t}}{\left(1-e^{-t}\right)^{d}}=(-1)^{d} \sum_{n=0}^{\infty} \frac{(-t)^{n-d}}{n !} B_{n}^{(d)}(a)
$$

We use the residue theorem to see that

$$
\operatorname{Res}_{s=z} \zeta_{\mathcal{B}}(s, a)=\frac{(-1)^{d+z}}{(z-1) !(d-z) !} B_{d-z}^{(d)}(a)
$$

for $z=1, \ldots, d$. The needed leading poles are

$$
\begin{aligned}
\operatorname{Res}_{s=d} \zeta_{\mathcal{B}}(s, a) & =\frac{1}{(d-1) !} \\
\operatorname{Res}_{s=d-1} \zeta_{\mathcal{B}}(s, a) & =\frac{d-2 a}{2(d-2) !} .
\end{aligned}
$$


This shows

$$
\begin{aligned}
\operatorname{Res} B_{0}(d-1 ; P, A) & =\frac{1}{2} d_{s} \epsilon \frac{\Gamma\left(\frac{m}{2}\right)}{\sqrt{\pi} \Gamma\left(\frac{m+1}{2}\right)(m-2) !}, \\
\operatorname{Res} B_{0}(d-2 ; P, A) & =\frac{1}{4} d_{s} \epsilon \frac{\Gamma\left(\frac{m-1}{2}\right)(m-3)}{\sqrt{\pi} \Gamma\left(\frac{m}{2}\right)(m-2) !}, \\
\operatorname{Res} B_{-1}(d-2 ; P, A) & =-\frac{1}{4} d_{s} \epsilon \frac{(m-3)}{(m-2) !},
\end{aligned}
$$

and these are all the terms contributing to the residues of $\eta$ at $s=d-1$ and $s=d-2$. Comparing with (5.d) and (5.e), after suitable rearrangements of the $\Gamma$-function [24, we use the doubling formula

$$
\Gamma(2 x)=\frac{2^{2 x-1}}{\sqrt{\pi}} \Gamma(x) \Gamma\left(x+\frac{1}{2}\right),
$$

and $\Gamma(x+1)=x \Gamma(x)$, we read off

$$
\begin{aligned}
c_{m}^{2} & =-\frac{1}{4} \beta(m), \\
c_{m}^{16} & =\frac{1}{2(m-2)}\left(1-\frac{1}{2} \pi(m-1) \beta(m)\right) .
\end{aligned}
$$

This completes the proof of Theorem 1.2

\section{ACKNOWLEDGEMENTS}

Research of PG was partially supported by the MPI (Leipzig, Germany). KK acknowledges support by the Baylor University Summer Sabbatical Program, by the Baylor University Research Committee, and by the MPI (Leipzig, Germany). Research of JHP was supported by Korea Science and Engineering Foundation Grant (R05-2003-000-10884-0).

\section{REFERENCES}

[1] M. Abramowitz and I.A. Stegun, Handbook of mathematical function, Natl. Bur. Stand. Appl. Math. Ser. 55 [U.S. GPO, Washington, D.C.] [Dover, New York, reprinted 1972].

[2] P. Amsterdamski, A. Berkin, and D. O'Connor, $b_{8}$ Hamidew coefficient for a scalar field, Classical Quantum Gravity 6 (1989), 1981-1991.

[3] M. F. Atiyah, V. K. Patodi, and I. M. Singer, Spectral asymmetry and Riemannian geometry I, Math. Proc. Cambridge Philos. Soc. 77 (1975), 43-69; II 78 (1975), 405-432; III 79 (1976), 71-79.

[4] I. G. Avramidi, The covariant technique for the calculation of the heat kernel asymptotic expansion, Phys. Lett. B 238 (1990), 92-97.

[5] R. Ball and H. Osborn, Large mass expansions for one loop effective actions and fermion currents, Nucl. Phys. B263 (1986) 245-264.

[6] E.W. Barnes, On the Theory of the Multiple Gamma Function, Trans. Camb. Phil. Soc. 19 (1903), 374-425.

[7] C.G. Beneventano, P. Gilkey, K. Kirsten, and E. M. Santangelo, Strong ellipticity and spectral properties of chiral bag boundary conditions, J. Phys A: Math. Gen. 36 (2003), 11533-11543.

[8] C. G. Beneventano, E. M. Santangelo, and A. Wipf, Spectral asymmetry for bag boundary conditions, J. Phys. A: Math. Gen. 35 (2002), 9343-9354.

[9] M. Bordag, E. Elizalde and K. Kirsten, Heat kernel coefficients of the Laplace operator on the D-dimensional ball, J. Math. Phys. 37 (1996), 895-916.

[10] M. Bordag, K. Kirsten and S. Dowker, Heat-kernels and functional determinants on the generalized cone, Commun. Math. Phys. 182 (1996), 371-394.

[11] T. Branson and P. Gilkey, Residues of the eta function for an operator of Dirac type, J. Funct. Anal. 108 (1992), 47-87.

[12] T. Branson and P. Gilkey, Residues of the eta function for an operator of Dirac type with local boundary conditions, Diff. Geo. and its Appl. 2 (1992), 249-267.

[13] R. Camporesi and A. Higuchi, On the eigenfunctions of the Dirac operator on spheres and real hyperbolic spaces, J. Geom. Phys. 20 (1996), 1-18. 
[14] P.D. D'Eath and G. Esposito, Spectral boundary conditions in one-loop quantum cosmology, Phys. Rev. D 44 (1991) 1713-1721.

[15] J.S. Dowker and G. Kennedy, Finite temperature and boundary effects in static spacetimes, J. Phys. A: Math. Gen. 11 (1978) 895-920.

[16] J.S. Dowker, P.B. Gilkey, and K. Kirsten, Heat asymptotics with spectral boundary conditions, Geometric Aspects of Partial Differential Equations, Contemporary Mathematics 242 (1999) AMS, 107-124.

[17] E. Elizalde, S.D. Odintsov, A. Romeo, A.A. Bytsenko and S. Zerbini, Zeta regularization techniques with applications, World Scientific, Singapore, 1994.

[18] G. Esposito, Quantum Gravity, Quantum Cosmology and Lorentzian Geometries, Lecture Notes in Physics m12, Springer-Verlag, Berlin, 1994.

[19] P. Forgacs, L. O'Raifeartaigh and A. Wipf, Scattering theory, U(1) anomaly and index theorems for compact and non-compact manifolds, Nucl. Phys. B293 (1987) 559-592.

[20] P. Gilkey, The spectral geometry of a Riemannian manifold, J. Differential Geom. 10 (1975), 601-618.

[21] P. Gilkey, Invariance Theory, the Heat Equation, and the Atiyah-Singer Index theorem, ( $2^{\text {nd }}$ edition), CRC Press [Boca Raton, Florida; ISBN 0-8493-7874-4] (1994).

[22] P. Gilkey, Asymptotic formulae in spectral geometry, CRC Press (2004), ISBN 1-58488$358-8$.

[23] P. Gilkey and K. Kirsten, Heat asymptotics with spectral boundary conditions II, Proc. Royal Society of Edinburgh 133A (2003), 333-361.

[24] I.S. Gradshteyn and I.M. Ryzhik, Tables of Integrals, Series and Products, Academic Press [New York] (1965).

[25] G. Grubb and R. Seeley, Weakly parametric pseudodifferential operators and Atiyah Patodi Singer boundary problems, Invent. Math. 121 (1995), 481-529.

[26] - Zeta and eta functions for Atiyah-Patodi-Singer operators, J. Geo. Anal. 6 (1996), $31-77$.

[27] M. Hortaçsu, K.D. Rothe and B. Schroer, Zero-energy eigenstates for the Dirac boundary problem, Nucl. Phys. B171 (1980) 530-542.

[28] R. Jackiw and C. Rebbi, Conformal properties of a Yang-Mills pseudoparticle, Phys. Rev. D 14 (1976) 517-523.

[29] K. Kirsten, Spectral Functions in Mathematics and Physics, Chapman \& Hall/CRC Press, Boca Raton, 2001.

[30] J. Lott, Eta and torsion, Les Houches, Session LXIV, 1995, Quantum Symmetries, Eds. A. Connes, K. Gawẹdzki and J. Zinn-Justin, Elsevier Science B.V., 1998, p. 947-955.

[31] J. Lott, Vacuum charge and the eta function, Commun. Math. Phys. 93 (1984) 533-558.

[32] H. P. McKean and I. M. Singer, Curvature and the eigenvalues of the Laplacian, J. Differential Geom. 1 (1967), 43-69.

[33] V. Moretti, Comments on the stress-energy tensor operator in curved spacetime, Commun. Math. Phys. 232 (2003) 189-221.

[34] A.J. Niemi and G.W. Semenoff, Index theorems on open infinite manifolds, Nucl. Phys. B269 (1986) 131-169.

[35] A.J. Niemi and G.W. Semenoff, Axial-anomaly-induced fermion fractionization and effective gauge-theory actions in odd-dimensional space-times, Phys. Rev. Lett. 51 (1983) 2077.

[36] A.J. Niemi and G.W. Semenoff, Fractional fermion number at finite temperature, Phys. Lett. B 135 (1984) 121-124.

[37] A.J. Niemi and G.W. Semenoff, Fermion number fractionization in quantum field theory, Phys. Rep. 135 (1986) 99-193.

[38] N.E. Norlund, Mémoire sur les polynomes de Bernoulli, Acta Math. 43 (1922), 121-196.

[39] M. Paranjape and G. Semenoff, Spectral asymmetry, trace identities and the fractional fermion number of magnetic monopoles, Phys. Lett. B 132 (1983) 369-373.

[40] K. Schleich, Semiclassical wave function of the Universe at small three-geometries, Phys. Rev. D 32 (1985) 1889-1898.

[41] R.T. Seeley, Complex powers of an elliptic operator, Singular Integrals, Chicago 1966. Proc. Sympos. Pure Math. 10 (1968) 288-307, American Mathematics Society, Providence, RI.

[42] G. 't Hooft, Computation of the quantum effects due to a four-dimensional pseudoparticle, Phys. Rev. D 14 (1976) 3432-3450.

[43] C. Wiesendanger and A. Wipf, Running coupling constants from finite size effects, Ann. Phys. 233 (1994) 125-161.

PG: Math. Dept., University of Oregon, Eugene, Or 97403, USA

E-mail address: gilkey@darkwing.uoregon.edu 
KK: Department of Mathematics, Baylor University, Waco, TX 76798, USA

E-mail address: Klaus_Kirsten@baylor.edu

JHP: Dept. of Computer Engineering, Honam University, Gwanguu 506-714 Korea

E-mail address: jhpark@honam.ac.kr 